\title{
The ESR intensity and the Dzyaloshinsky-Moriya interaction of the nanoscale molecular magnet $V_{15}$
}

\author{
Manabu Machida,, ,* Toshiaki Iitaka, ${ }^{2,+1}$ and Seiji Miyashita ${ }^{3, t+}$ \\ ${ }^{1}$ Department of Mathematics, University of Michigan, \\ 530 Church Street, Ann Arbor, MI 48109, USA \\ ${ }^{2}$ Computational Astrophysics Laboratory, \\ RIKEN (The Institute of Physical and Chemical Research), \\ 2-1 Hirosawa, Wako, Saitama 351-0198, Japan \\ ${ }^{3}$ Department of Physics, Graduate School of Science, \\ The University of Tokyo, 7-3-1 Hongo, \\ Bunkyo-ku, Tokyo 113-0033, Japan
}

(Dated: November 6, 2018)

\begin{abstract}
The intensity of electron spin resonance (ESR) of the nanoscale molecular magnet $\mathrm{V}_{15}$ is studied. We calculate the temperature dependence of the intensity at temperatures from high to low. In particular, we find that the low-temperature ESR intensity is significantly affected by the Dzyaloshinsky-Moriya interaction.
\end{abstract}




\section{INTRODUCTION}

The $\mathrm{V}_{15}$ molecule has been one of promising nanometer-scale molecular magnets since it was first synthesized. $\underline{\underline{1}}^{-\underline{5}}$ It is the complex of formula $\mathrm{K}_{6}\left[\mathrm{~V}_{15}^{\mathrm{IV}} \mathrm{As}_{6} \mathrm{O}_{42}\left(\mathrm{H}_{2} \mathrm{O}\right)\right] \cdot 8 \mathrm{H}_{2} \mathrm{O}$. In $\mathrm{V}_{15}$, fifteen vanadium ions of spin $1 / 2$ form almost a sphere. Three spins in the middle are sandwiched by the upper and lower hexagons.

Different experiments on the magnetization process have shown that the magnetization changed adiabatically in a fast sweeping field, and a magnetic plateau appeared in a slow sweeping field due to thermal bath attached to the molecule $\underline{6}^{-\underline{\underline{8}}}$ The latter phenomenon, which is called the phonon bottleneck effect, is theoretically analyzed from a general point of view of the magnetic Foehn effect. $\underline{\underline{9}}$ This smooth change of the magnetization at $H=0 \mathrm{~T}$ implies the existence of an avoided-level-crossing energy structure. The structure of avoided level crossing has been studied. In a model of the triangle Heisenberg antiferromagnet with three spins, at $H=0$, two sets of $S=1 / 2$ doublets overlap, and 4 states degenerate. The degeneracy is resolved into two sets of Kramers doublets by perturbation such as anisotropy, Dzyaloshinsky-Moriya interaction (DMI) $\stackrel{\underline{10}}{\underline{13}} \underline{\underline{13}}$ and also the hyperfine interaction. $\underline{\underline{14}}$ Depending on the type of perturbation, there appear many kinds of energy structure. At the crossing point of the two states of $m=1 / 2$ of the doublet states and one of the quartet state $(m=3 / 2), H=H_{\mathrm{c}}$, a kind of avoided level crossing is formed. It has been pointed out that adiabatic change causes the change of magnetization from $m-1 / 2$ to $m=1$ because one state adiabatically changes to the state with $m=3 / 2 . \underline{15}, 16$ In $\mathrm{V}_{15}$, the equilibrium magnetization curve shows smooth change at zero field from $1 / 2$ to $1 / 2$ and at $2.8 \mathrm{~T}$ ( $\equiv H_{\mathrm{c}}$ ) from $1 / 2$ to $3 / 2$. $\mathrm{V}_{15}$ can be described by a triangle model but details of the DMI in $\mathrm{V}_{15}$ are not yet fully understood.

In this paper, first we numerically calculate the temperature dependence of the ESR intensity of $\mathrm{V}_{15}$ using a new numerical method (the double Chebyshev polynomial method) of calculating the Kubo formula. We find that the model Hamiltonian for $\mathrm{V}_{15}$ including the DMI successfully reproduces the experimental temperature dependence of the ESR intensity. Second we investigate the ESR at very low temperatures. We find that the intensity ratio (the intensity of $\mathrm{V}_{15}$ divided by that of a spin $1 / 2$ ) is affected by the DMI at small fields. We propose that experimental observation of the intensity ratio enables us to estimate the DMI in $\mathrm{V}_{15}$. Finally, we analyze the ESR at low temperatures using a triangle model whose 
energy levels model the low-lying levels of $\mathrm{V}_{15}$.

\section{MODEL AND FORMULATION}

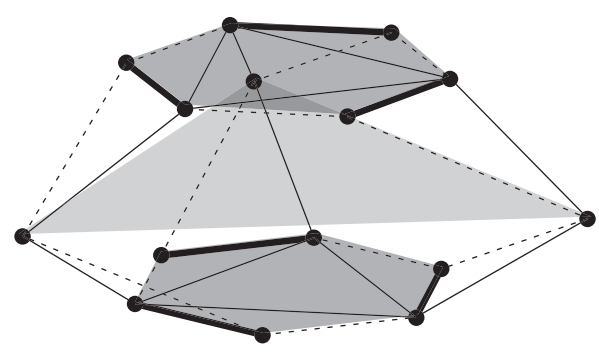

$\begin{array}{ll}J & \longrightarrow \\ J_{1} & \cdots \cdots \cdots \\ J_{2} & \end{array}$

FIG. 1: Schematic picture of $\mathrm{V}_{15}$.

Figure 1 shows the structure of vanadium ions in $\mathrm{V}_{15}$. We consider the following spin Hamiltonian for $\mathrm{V}_{15} \cdot \underline{17-19}$

$$
\mathcal{H}=-\sum_{\langle i, j\rangle} J_{i j} \boldsymbol{S}_{i} \cdot \boldsymbol{S}_{j}+\sum_{\langle i, j\rangle} \boldsymbol{D}_{i j} \cdot\left[\boldsymbol{S}_{i} \times \boldsymbol{S}_{j}\right]-\sum_{i} \boldsymbol{H} \cdot \boldsymbol{S}_{i}
$$

The first term on the right-hand side of Eq. (1) describes the Heisenberg interaction. Coefficients $J_{i j}$ take three values $J, J_{1}$, and $J_{2}\left(|J|>\left|J_{2}\right|>\left|J_{1}\right|\right)$ depending on the bonds on the upper and lower hexagons. Three spins between two hexagons interact with the hexagons by $J_{1}$ and $J_{2}$. There is no interaction among these three spins ${ }^{3}$. We set $J=-800 \mathrm{~K}$, $J_{2}=-350 \mathrm{~K}$, and $J_{1}=-225 \mathrm{~K} . \underline{20}$ The second term describes the DMI. We assume the existence of DM vectors $\left\{\boldsymbol{D}_{i j}\right\}$ at the bonds of $J$. In the third term, $\boldsymbol{H}$ denotes the static magnetic field applied to the molecule. We will ignore other effects such as dipolar fields, hyperfine interactions, and the crystal field, which are considered to be negligibly small. Indeed, the dipolar and hyperfine fields are estimated as $1 \mathrm{mK}$ and $50 \mathrm{mK}$, respectively ${ }^{\underline{7}}$. 

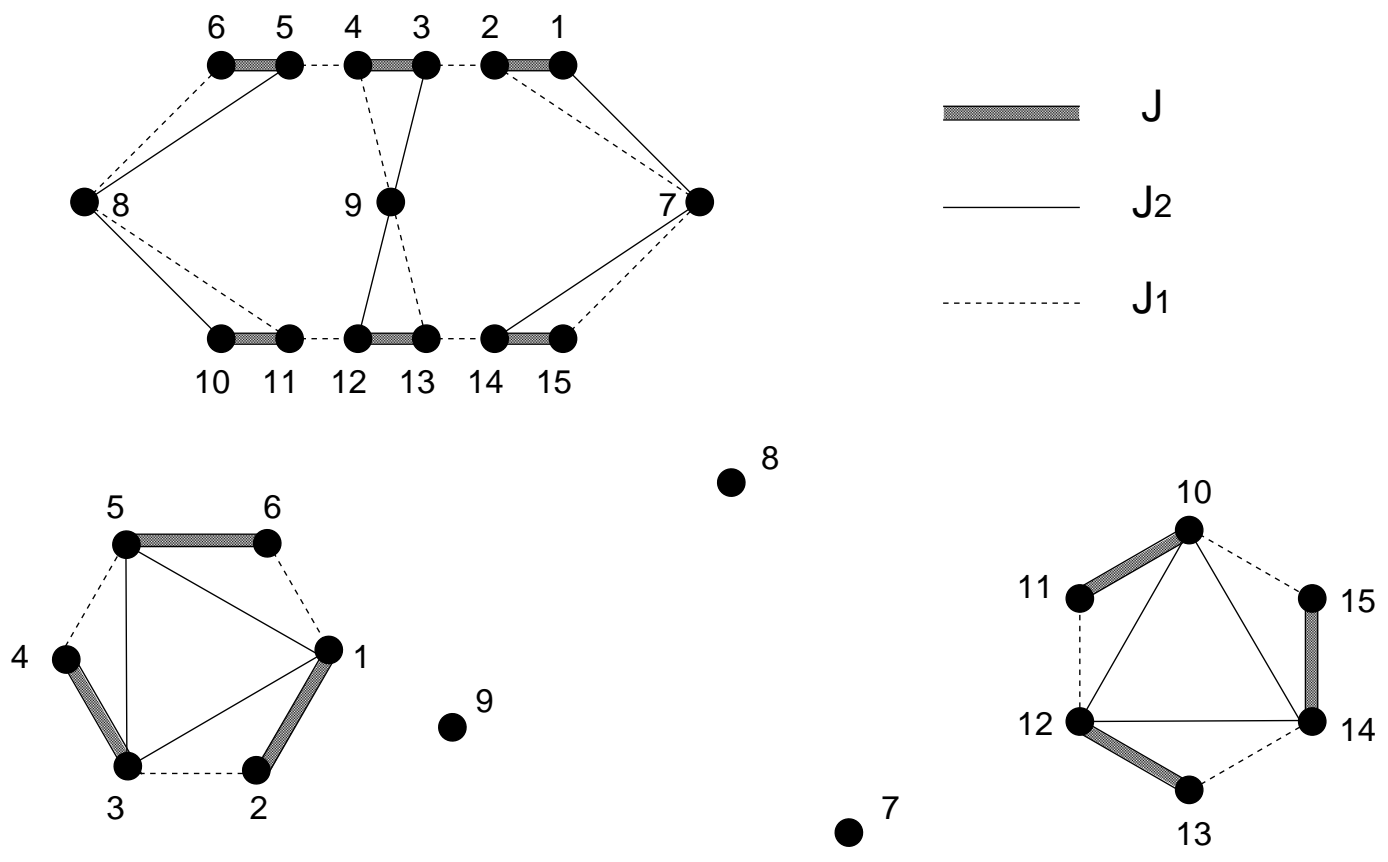

FIG. 2: Heisenberg interactions between the spins.

Figure 2 explains the interactions between spins. If we assume the $D_{3}$ symmetry of $\mathrm{V}_{15}$, the lower hexagon differs from the upper hexagon by rotation $\pi / 6$ and we have only one free DM vector, say $\boldsymbol{D}_{1,2}$ (Fig. (3). We take the vector $\boldsymbol{D}_{1,2}$ to be $D_{1,2}^{x}=D_{1,2}^{y}=D_{1,2}^{z}=40 \mathrm{~K}$. Let us define

$$
\hat{R}(\theta)=\left(\begin{array}{ccc}
\cos \theta & -\sin \theta & 0 \\
\sin \theta & \cos \theta & 0 \\
0 & 0 & 1
\end{array}\right), \quad \hat{P}=\left(\begin{array}{ccc}
-1 & 0 & 0 \\
0 & 1 & 0 \\
0 & 0 & -1
\end{array}\right) .
$$

We obtain the other DM vectors on the upper hexagon by rotating $\boldsymbol{D}_{1,2}$ by $2 \pi / 3$ and $4 \pi / 3$ :

$$
\boldsymbol{D}_{3,4}=\hat{R}\left(\frac{4 \pi}{3}\right) \boldsymbol{D}_{1,2}, \quad \boldsymbol{D}_{5,6}=\hat{R}\left(\frac{2 \pi}{3}\right) \boldsymbol{D}_{1,2},
$$

i.e., $D_{3,4}^{x}=14.641 \mathrm{~K}, D_{3,4}^{y}=-54.641 \mathrm{~K}, D_{3,4}^{z}=40 \mathrm{~K}, D_{5,6}^{x}=-54.641 \mathrm{~K}, D_{5,6}^{y}=14.641 \mathrm{~K}$, and $D_{5,6}^{z}=40 \mathrm{~K}$. The DM vectors on the lower hexagon are obtained as

$$
\boldsymbol{D}_{10,11}=\hat{P} \boldsymbol{D}_{1,2}, \quad \boldsymbol{D}_{12,13}=\hat{R}\left(\frac{2 \pi}{3}\right) \boldsymbol{D}_{10,11}, \quad \boldsymbol{D}_{14,15}=\hat{R}\left(\frac{4 \pi}{3}\right) \boldsymbol{D}_{10,11},
$$

i.e., $D_{10,11}^{x}=-40 \mathrm{~K}, D_{10,11}^{y}=40 \mathrm{~K}, D_{10,11}^{z}=-40 \mathrm{~K}, D_{12,13}^{x}=-14.641 \mathrm{~K}, D_{12,13}^{y}=-54.641 \mathrm{~K}$, $D_{12,13}^{z}=-40 \mathrm{~K}, D_{14,15}^{x}=54.641 \mathrm{~K}, D_{14,15}^{y}=14.641 \mathrm{~K}$, and $D_{14,15}^{z}=-40 \mathrm{~K}$. 
Let us calculate the ESR intensity of $\mathrm{V}_{15}$ using the Hamiltonian (1). By the Kubo formula 21,22 , the imaginary part of the susceptibility $\chi^{\prime \prime}(\omega, T)$ on the transverse field $\boldsymbol{H}$ parallel to the $x$-axis is given by the Fourier transform of the spin-spin correlation function;

$$
\chi^{\prime \prime}(\omega ; T)=\left(1-\mathrm{e}^{-\beta \omega}\right) \operatorname{Re} \int_{0}^{\infty}\left\langle M^{x} M^{x}(t)\right\rangle \mathrm{e}^{-\mathrm{i} \omega t} \mathrm{~d} t
$$

where $\omega$ is the frequency of the radiation field, $T$ is temperature, and $\beta=1 / T$. The absorption is given by

$$
I(\omega ; T)=\frac{\omega H_{\mathrm{R}}^{2}}{2} \chi^{\prime \prime}(\omega ; T),
$$

where $H_{\mathrm{R}}$ is the amplitude of the radiation field. We obtain the ESR intensity $I(T)$ by integrating $I(\omega, T)$ with respect to $\omega$;

$$
I(T)=\int_{0}^{\infty} I(\omega, T) \mathrm{d} \omega
$$

In the present paper, we obtain $I(T)$ without diagonalization. Our method can readily be applied to other nanomagnets. In Appendix A, we describe the numerical method of calculating the Kubo formula for huge Hamiltonian quantum many-body systems. ${ }^{19}$ This method, which we call the double Chebyshev expansion method (DCEM), realizes $O(N)$ calculation in both speed and memory. In the method, we evaluate the summation over all the states in the expression of the correlation function by using the average over a few number of random initial states. Furthermore, we calculate the exponential operators $\mathrm{e}^{-\beta \mathcal{H}}$ and $\mathrm{e}^{-\mathrm{i} \mathcal{H} t}$ by expanding them in the Chebyshev polynomial. The DCEM takes all the states in the Hilbert space into account and enables us to obtain the ESR at any temperature. 


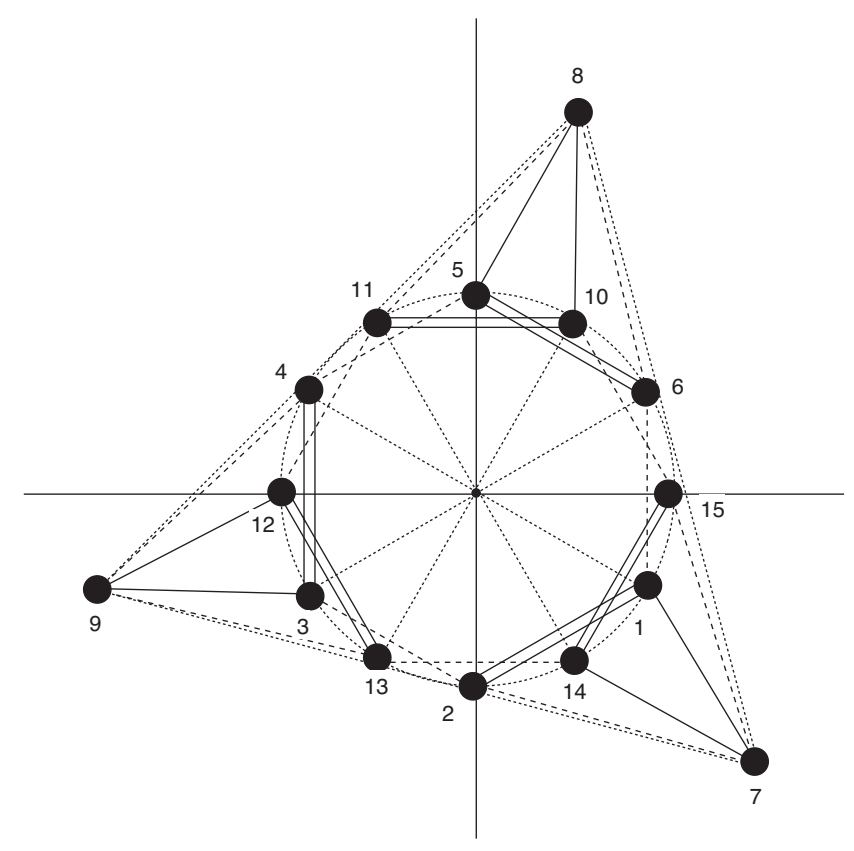

FIG. 3: The $D_{3}$ symmetry of $\mathrm{V}_{15}$.

\section{TEMPERATURE DEPENDENCE OF THE ESR INTENSITY}

We study the temperature dependence of the ESR intensity of $\mathrm{V}_{15}$. We apply the magnetic field parallel to the $c$-axis of the molecule ( $z$-axis): $\boldsymbol{H}=(0,0, H)$. Figure 4 shows intensities at $H=2 \mathrm{~T}$ from $1 \mathrm{~K}$ to $10000 \mathrm{~K}$. The intensity obtained by the DCEM (solid circles) is plotted together with experimental data by Ajiro, et al $\underline{\underline{23}}$ (solid squares). The intensity by the DCEM in Fig. 团agrees with the experimental data. In addition, the dashed line denotes the intensity

$$
I_{1}(T)=H \tanh \frac{\beta H}{2}
$$

of an isolated spin $1 / 2$. The short-dashed line, dotted line, and dash-dotted line denote $2 I_{1}(T), 3 I_{1}(T)$, and $15 I_{1}(T)$ for non-interacting 2,3 , and 15 spins, respectively. 


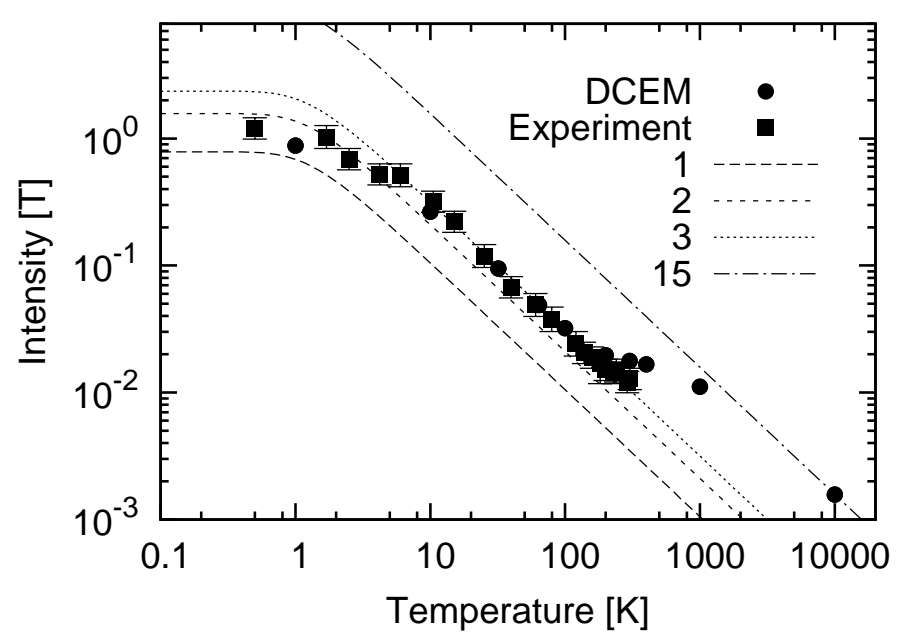

FIG. 4: The temperature dependences of the intensity for $\boldsymbol{H} \| z$ and $H=2 \mathrm{~T}$ and the experimental intensity.

When the temperature is sufficiently higher than the interactions, spins in $V_{15}$ are almost isolated. Therefore, the intensity is expected to meet the dash-dotted line at very high temperatures. In Fig. 4, the intensity starts to deviate from the dotted line near 200K. As the temperature decreases, the effective number of spins changes from 15 to 3 , and the intensity stays on the dotted line at temperatures between $10 \mathrm{~K}$ and $100 \mathrm{~K}$.

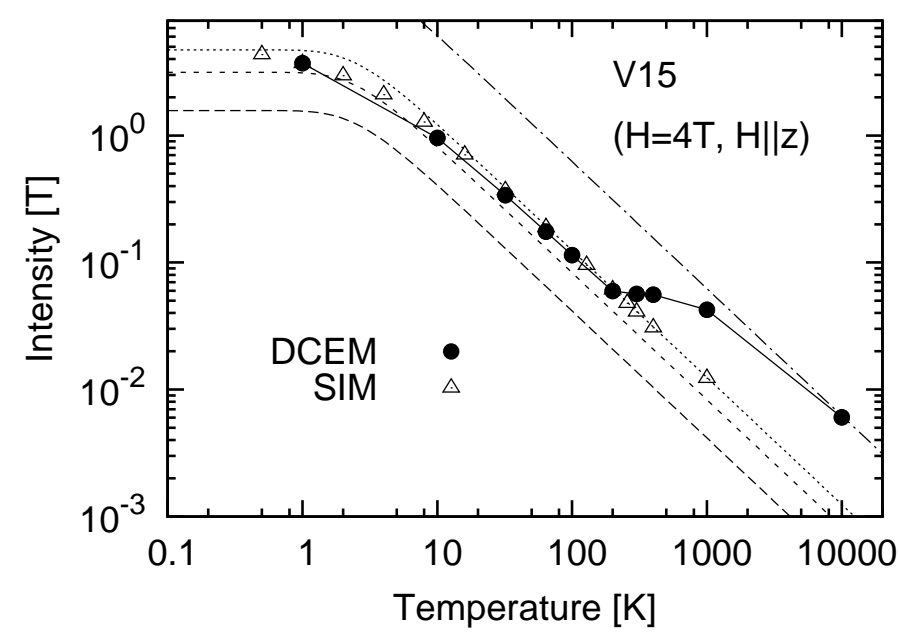

FIG. 5: The temperature dependence of the intensity for $\boldsymbol{H} \| z$ and $H=4 \mathrm{~T}$.

Figure 5 is the same as Fig. 4 except $H=4 \mathrm{~T}$. At temperatures above 10K, the intensity 
in Fig. [5 behaves almost the same as that in Fig. 4. However, the temperature dependence

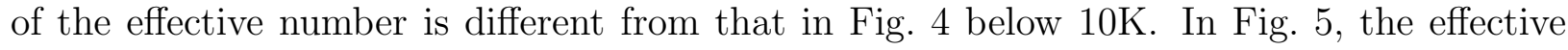
number of spins changes from 15 to 3 at high temperatures, and stays on the dotted line for $3 I_{1}(T)$ as temperature decreases. A similar behavior of the low-temperature intensity has also been predicted. $\underline{\underline{24}}$

\section{THE LOW-TEMPERATURE INTENSITY RATIO}

\section{A. $I(T)$ at low temperatures}

Although the DMI affects $I(T)$ only mildly at $T>1 \mathrm{~K}$, it significantly changes $I(T)$ at low temperatures. At low temperatures, only transitions among low-lying energy levels near the ground state are responsible for the ESR absorption. This fact enables us to obtain the intensity by investigating the transitions among the lowest eight levels. Thus, we can

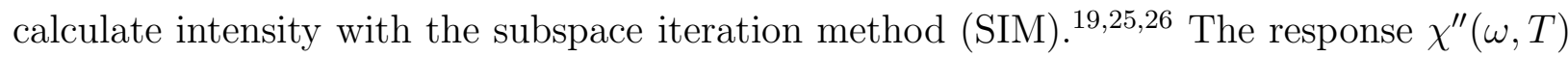
is obtained by direct diagonalization in the small reduced space. In Fig. 6, we compare the result of Fig. 4 and the data obtained by the SIM. We find a good agreement below 100K. Above this temperature, the lowest eight states are not enough to represent the system.

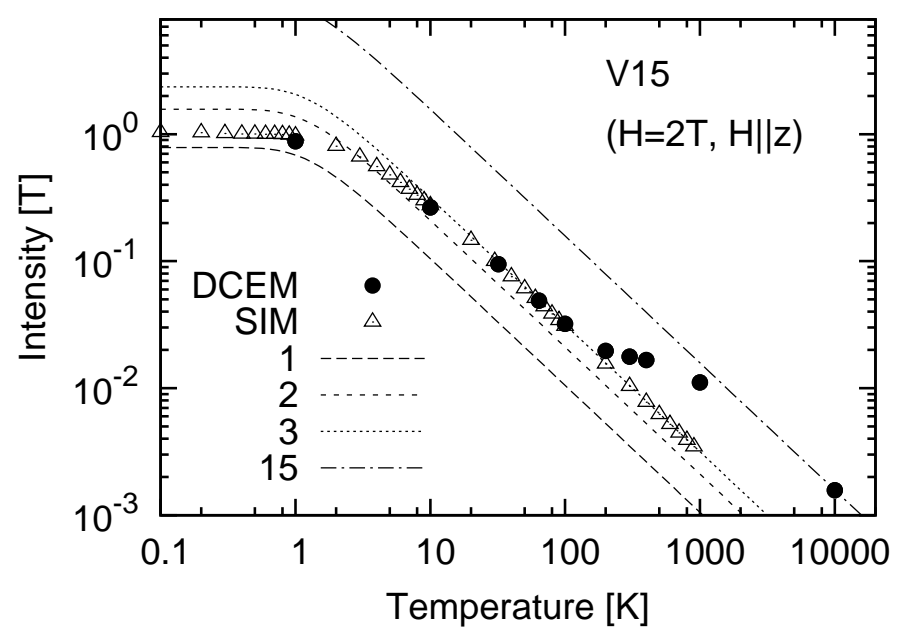

FIG. 6: The temperature dependence of the intensity for $\boldsymbol{H} \| z$ and $H=2 \mathrm{~T}$.

In Fig. $7(\mathrm{a})$, we show the lowest eight energy levels of $\mathrm{V}_{15}$ as a function of the magnetic field $H$ applied in the $z$-direction. Due to the DMI, we have an avoided level crossing near 
$3 \mathrm{~T}$ as shown in Fig. 7(b). Without the DMI, the gap closes and the two levels just cross each other.
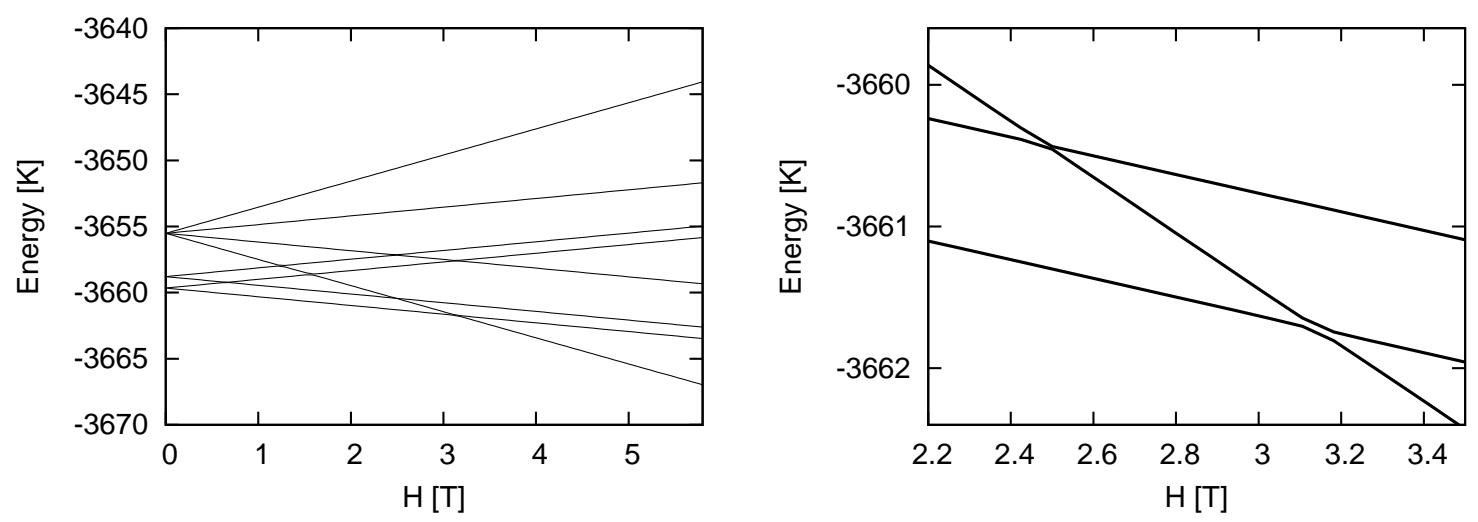

FIG. 7: (a) The lowest eight levels of $\mathrm{V}_{15}$ as a function of $\boldsymbol{H} \| z$. (b) Magnified figure near 3T.

We define the intensity ratio $R(T)$ as

$$
R(T)=I(T) / I_{1}(T)
$$

where $I_{1}(T)$ is the intensity of a single spin (Eq. (8) $)$. In the previous section, we have found $R(T) \simeq 3$ for $H=4 \mathrm{~T}$ and $R(T)$ is slightly larger than 1 for $H=2 \mathrm{~T}$ in the low temperature limit. Figure 8 shows $R(T)$ in a low temperature region. In Fig. 8, the circles and triangles show $R(T)$ at $H=57.8 \mathrm{GHz}(\simeq 2 \mathrm{~T})$ and at $H=108 \mathrm{GHz}(\simeq 4 \mathrm{~T})$, respectively. The dashed lines in Fig. 8 show the ratios without the DMI at $H=2 \mathrm{~T}$ and at $H=4 \mathrm{~T}$ for comparison. First, we notice that $H_{\mathrm{c}}$ can be experimentally estimated with the low-temperature ESR by measuring the field at which the destination of the intensity changes. Second, we see that $R(T=0 \mathrm{~K})$ at $H=2 \mathrm{~T}$ deviates from 1 due to the $\mathrm{DMI}$, while $R(T=0 \mathrm{~K})$ at $H=4 \mathrm{~T}$ stays very close to the dotted line. Thus it would be possible to experimentally determine the DMI in $\mathrm{V}_{15}$ by observing the intensity at low temperatures. 


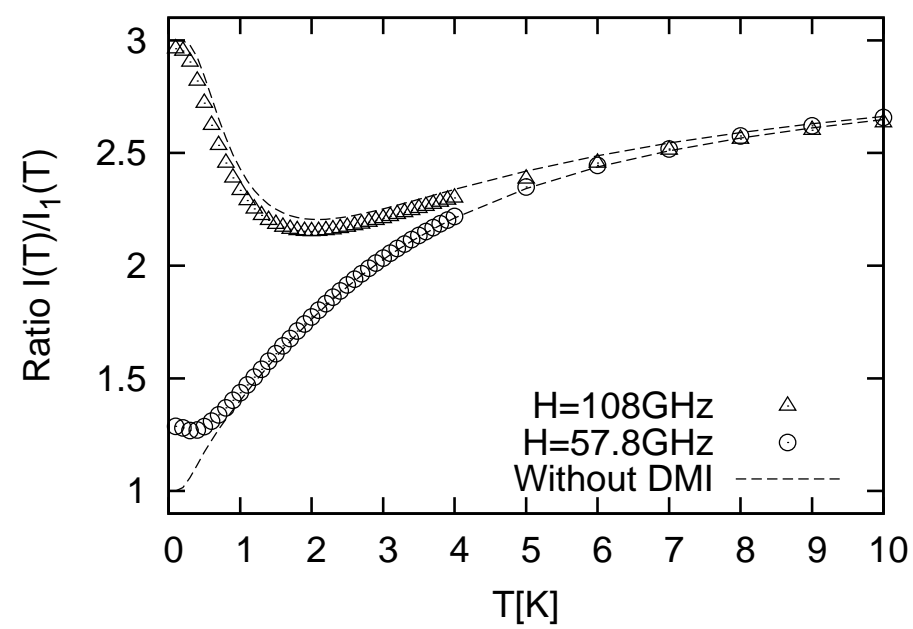

FIG. 8: The intensity ratios of $\mathrm{V}_{15}$ as a function of the temperature. The intensity ratios without the DMI are also shown for comparison.

Furthermore, we study intensity ratios at various fields near the avoided level crossing point (hereafter we refer to this field as $H_{\mathrm{c}}$ ) in the presence (Fig. 9(a)) and absence (Fig. 9(b)) of the DMI. The ratio goes to 3 as $T \rightarrow 0$ for a field higher than $H_{\mathrm{c}}$. In contrast, it goes near 1 for a lower field. The derivative of the line for $H=2 \mathrm{~T}$ in Fig. 9(a) is negative at $0 \mathrm{~K}$, while that for $H=3 \mathrm{~T}$ is positive. Hence the line for $H=2 \mathrm{~T}$ has a dip at $T \simeq 0.5 \mathrm{~K}$. In the absence of the DMI, the line for $H=2 \mathrm{~T}$ does not have a dip.
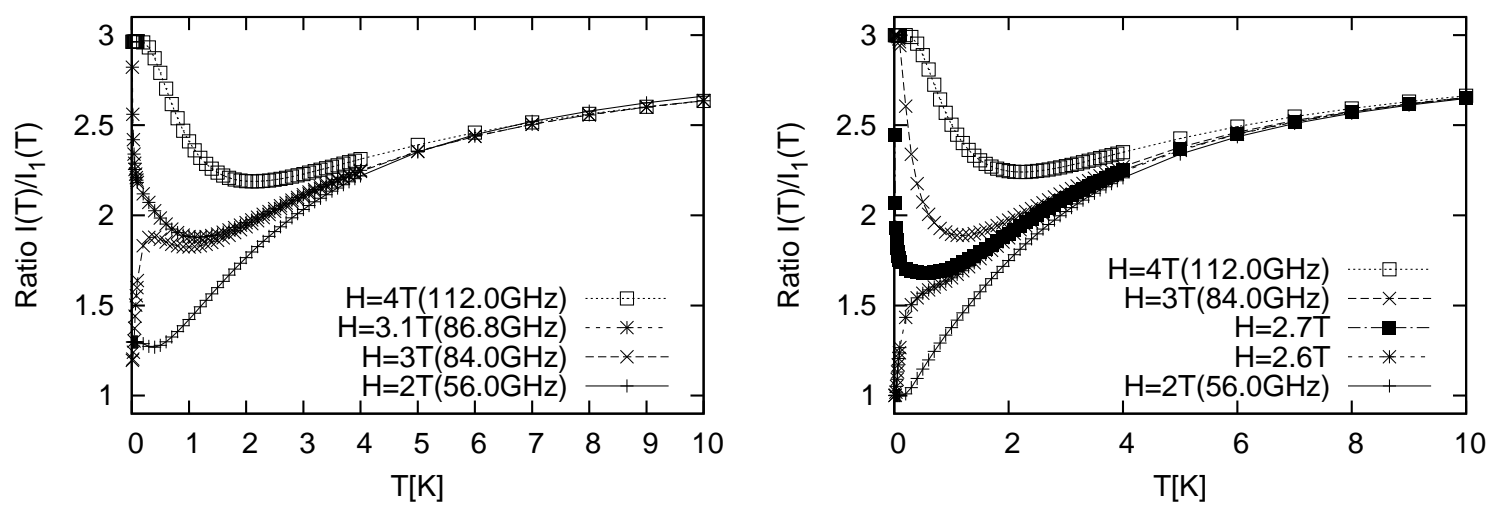

FIG. 9: (a) Intensity ratios of $\mathrm{V}_{15}$ as a function of temperature for various fields. (b) Intensity ratios without the DMI. 


\section{B. Triangle Model}

As shown in Figs. 8 and 9(a), the intensity ratio for lower fields deviates from 1 in the low-temperature limit. Here, we study the mechanism of this deviation analytically in a triangle model of three spins which well describes the low-lying energy structure of $\mathrm{V}_{15}$.

The triangle model 18 is given by the Hamiltonian Eq. (11) with $J_{i j}=J<0, \boldsymbol{H}=(0,0, H)$, $H>0$,

$$
\begin{array}{ll}
D_{12}^{x}=D_{x}, & D_{12}^{y}=D_{y}, \\
D_{23}^{x}=\left(-D_{x}+\sqrt{3} D_{y}\right) / 2, & D_{23}^{y}=\left(-\sqrt{3} D_{x}-D_{y}\right) / 2, \\
D_{31}^{x}=\left(-D_{x}-\sqrt{3} D_{y}\right) / 2, & D_{31}^{y}=\left(\sqrt{3} D_{x}-D_{y}\right) / 2,
\end{array}
$$

and

$$
D_{12}^{z}=D_{23}^{z}=D_{31}^{z}=D_{z}
$$

Note that the elements of the DM vectors are chosen so that the model satisfies the $C_{3}$ symmetry. If we put $J=-2.5 \mathrm{~K}$ and $D_{x}=D_{y}=D_{z}=0.25 \mathrm{~K}(\equiv D)$, energy levels of the triangular model well reproduce the lowest eight levels of $\mathrm{V}_{15}$ as shown in Fig. 10, In the absence of the DMI, the ground state magnetization $M^{z}$ changes from $1 / 2$ to $3 / 2$ at

$$
H_{\mathrm{c}} \equiv \frac{3}{2}|J| \simeq 2.8 \mathrm{~T}
$$

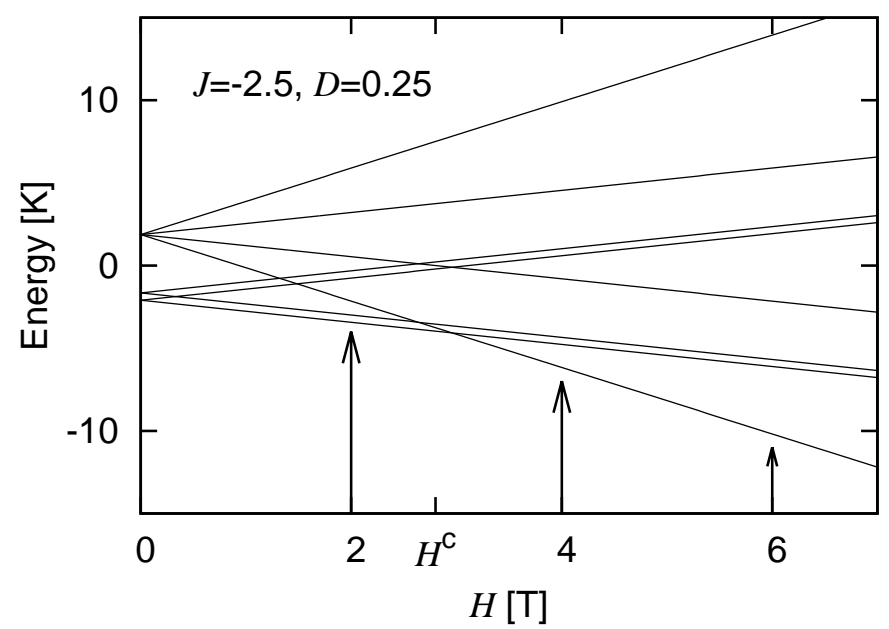

FIG. 10: Energy levels of the triangle model as a function of the field $H$.

The intensity ratio $R_{3}(T)$ of this triangle model is obtained as 


$$
\begin{aligned}
R_{3}(T) & =\left[I_{1}(T) \sum_{m} \mathrm{e}^{-\beta E_{m}}\right]^{-1} \sum_{m} r(m) \\
r(m) & =\sum_{n\left(E_{n}>E_{m}\right)}\left(E_{n}-E_{m}\right)\left(\mathrm{e}^{-\beta E_{m}}-\mathrm{e}^{-\beta E_{n}}\right)\left|2\left\langle w_{m}\left|M_{x}\right| w_{n}\right\rangle\right|^{2},
\end{aligned}
$$

where $\left\{E_{m}\right\}$ and $\left\{\left|w_{m}\right\rangle\right\}$ are the eigenvalues and eigenvectors of the triangle-model Hamiltonian, respectively. In Appendix $\mathrm{B}, R_{3}(T)$ at low temperatures around $H_{\mathrm{c}}$ is calculated.

Let us explore the nonmonotonic temperature dependence of the intensities shown in Fig. 9 by using the triangle model with different values of $D_{x}, D_{y}$, and $D_{z}$.

First, we consider how the DMI causes the dip at $T \simeq 0.5 \mathrm{~K}$ for $H=4 \mathrm{~T}$ in Fig. 9)(a). In the absence of the DMI (Fig. 9(b)), by setting $D_{x}=D_{y}=D_{z}=0$, we obtain $\mathrm{d} R_{3}(T) / \mathrm{d} T>0$ for $H<H_{\mathrm{c}}$ and $\mathrm{d} R_{3}(T) / \mathrm{d} T<0$ for $H_{\mathrm{c}}<H$ (see Eq. (B15) in Appendix (B)). To investigate the intensities in Fig. 9(a), we set $D_{x}=D_{y}=0, D_{z} \neq 0$ (uniaxial). Note that the structure of the energy levels with $D_{x}=D_{y}=D_{z} \neq 0$ is the same as that in the uniaxial case only except that the gap closes in the uniaxial case. We find that $\mathrm{d} R_{3}(T) / \mathrm{d} T<0$ for $H \lesssim H_{\mathrm{c}}$ and $\mathrm{d} R_{3}(T) / \mathrm{d} T>0$ for $H \simeq H_{\mathrm{c}}$ (see Eq. (B17) in Appendix B), which implies a dip at a low field.

Next, let us consider the effect of a weak DMI. We set $D_{x}=D_{y}=D_{z}=D \neq 0$. By ignoring smaller terms than $O(D)$, we have (Appendix $\mathrm{B}$ )

$$
R_{3}(T) \simeq \begin{cases}1+\frac{\Delta}{H}-\frac{2 \Delta}{H} \mathrm{e}^{-\Delta / T} & \left(H<H_{\mathrm{c}}\right) \\ 3-\left(2-\frac{\Delta}{H}\right) \mathrm{e}^{-\left|H-H_{\mathrm{c}}-\Delta / 2\right| / T} & \left(H_{\mathrm{c}}<H\right)\end{cases}
$$

where we defined

$$
\Delta \equiv \sqrt{3} D
$$

Taking the limit $T \rightarrow 0$, we have

$$
R_{3}(T) \simeq \begin{cases}1+\frac{\Delta}{H} & \left(H<H_{\mathrm{c}}\right) \\ 3 & \left(H_{\mathrm{c}}<H\right)\end{cases}
$$

This term $\Delta / H$ describes the deviation of $R(T)$ from 1 , and the deviation is proportional to $D$.

\section{SUMMARY}

In this paper, we studied the ESR of the nanomagnet $\mathrm{V}_{15}$. We investigated the temperature dependence of the intensity on the DMI for different values of the static field $H$. The 
DMI significantly affects the low-temperature intensity of $\mathrm{V}_{15}$. We found that the intensity at $H<H_{\mathrm{c}}$ has a dip as a function of temperature due to the DMI. We analyzed the dip using the three-spin model and obtained analytical expressions of the intensity. Experimental measurement of the dip may be used to estimate the DMI of $\mathrm{V}_{15}$.

\section{Acknowledgments}

The authors would like to thank Professor Hans De Raedt for valuable discussions. The simulations were partially carried out by using the computational facilities of the Super Computer Center of Institute for Solid State Physics, The University of Tokyo, and the Advanced Center for Computing and Communication, RIKEN (The Institute of Physical and Chemical Research).

\section{Appendix A: DCEM}

The DCEM is an extension of the Boltzmann-weighted time-dependent method (BWTDM) $\stackrel{27}{\underline{2}, 28}$ The DCEM differs from the BWTDM by the third step below. The procedure of the DCEM is divided by the following five steps.

At the first step, we prepare a random vector $|\Phi\rangle \underline{\underline{29}} \underline{30}$ For a given basis $|n\rangle$ of the Hilbert space, this random vector is given by $|\Phi\rangle=\sum_{n=1}^{N}|n\rangle \mathrm{e}^{\mathrm{i} \theta_{n}}$. Here, the dimension of the Hilbert space is $N$ and the random numbers $\left\{\theta_{n}\right\}$ take values from $-\pi$ to $\pi$.

At the second step, we obtain the Boltzmann-weighted random vector $\left|\Phi_{\text {Boltz }}\right\rangle=$ $\mathrm{e}^{-\beta \mathcal{H} / 2}|\Phi\rangle$ by expanding it with the Chebyshev polynomial;

$$
\begin{aligned}
\mathrm{e}^{-\beta \mathcal{H} / 2} & =I_{0}(-\beta \Delta \lambda / 2) T_{0}\left(\mathcal{H}_{\mathrm{sc}}\right) \\
& +2 \sum_{k} I_{k}(-\beta \Delta \lambda / 2) T_{k}\left(\mathcal{H}_{\mathrm{sc}}\right),
\end{aligned}
$$

where $I_{k}(x)$ is the modified Bessel function and $T_{k}\left(\mathcal{H}_{\mathrm{sc}}\right)$ is the Chebyshev polynomial, which satisfies $T_{k}\left(\mathcal{H}_{\mathrm{sc}}\right)=2 \mathcal{H}_{\mathrm{sc}} T_{k-1}\left(\mathcal{H}_{\mathrm{sc}}\right)-T_{k-2}\left(\mathcal{H}_{\mathrm{sc}}\right), T_{0}\left(\mathcal{H}_{\mathrm{sc}}\right)=1$, and $T_{1}\left(\mathcal{H}_{\mathrm{sc}}\right)=\mathcal{H}_{\mathrm{sc}}$. We note that the eigenvalues of $\mathcal{H}_{\mathrm{sc}}(=\mathcal{H} / \Delta \lambda)$ are confined between -1 to 1 . By this procedure, we obtain vectors $\mathrm{e}^{-\beta \mathcal{H} / 2}|\Phi\rangle$ without diagonalization.

At the third step, we obtain $\left|\Phi_{\text {Boltz }} ; t\right\rangle=\mathrm{e}^{-\mathrm{i} \mathcal{H} t}\left|\Phi_{\text {Boltz }}\right\rangle$ and $\left|\Phi_{M^{x}} ; t\right\rangle=\mathrm{e}^{-\mathrm{i} \mathcal{H} t}\left|\Phi_{M^{x}}\right\rangle$, where $\left|\Phi_{M^{x}}\right\rangle=M^{x}\left|\Phi_{\text {Boltz }}\right\rangle$ and $M^{x}=\sum S_{j}^{x}$. In the BWTDM, the time evolution is performed by 
the leap frog method, which evolves a state $|\phi ; t\rangle$ as

$$
|\phi ; t+\Delta t\rangle=-2 \mathrm{i} \mathcal{H} \Delta t|\phi ; t\rangle+|\phi ; t-\Delta t\rangle .
$$

Note that the condition $E_{\max } \Delta t \ll 1$ should be satisfied, where $E_{\max }$ is the largest eigenvalue of the Hamiltonian. In the DCEM, we make use of the Chebyshev polynomial expansion in order to obtain the time evolution of the vector;

$$
\begin{aligned}
|\phi ; t+\tau\rangle & =\mathrm{e}^{-\mathrm{i} \tau \Delta \lambda \mathcal{H}_{\mathrm{sc}}}|\phi ; t\rangle \\
& =J_{0}(\tau \Delta \lambda) T_{0}\left(\mathcal{H}_{\mathrm{sc}}\right)|\phi ; t\rangle \\
& +2 \sum_{k}(-\mathrm{i})^{k} J_{k}(\tau \Delta \lambda) T_{k}\left(\mathcal{H}_{\mathrm{sc}}\right)|\phi ; t\rangle,
\end{aligned}
$$

where $J_{k}(x)$ is the Bessel function. Note that the time step $\tau$ is not necessarily small. In the ESR experiment for $\mathrm{V}_{15}$, the magnetic field $H(\sim 1 \mathrm{~K})$ is usually much smaller than the strongest coupling $|J|\left(\sim 10^{3} \mathrm{~K}\right)$. Hence the frequency of precession of the spins is small. This means that we need to evolve state vectors for a long time but do not need fine resolution of the time step. This is why the DCEM is more efficient than the BWTDM for the ESR of $\mathrm{V}_{15}$.

At the fourth step, we calculate the correlation function

$$
\begin{aligned}
\left\langle M^{x} M^{x}(t)\right\rangle & =\frac{\operatorname{Tre}^{-\beta \mathcal{H}} M^{x} \mathrm{e}^{\mathrm{i} \mathcal{H} t} M^{x} \mathrm{e}^{-\mathrm{i} \mathcal{H} t}}{\operatorname{Tre}^{-\beta \mathcal{H}}} \\
& =\frac{\left[\left\langle\Phi_{M^{x}} ; t\left|M^{x}\right| \Phi_{\text {Boltz }} ; t\right\rangle\right]_{\mathrm{av}}}{\left[\left\langle\Phi_{\text {Boltz }} \mid \Phi_{\text {Boltz }}\right\rangle\right]_{\mathrm{av}}},
\end{aligned}
$$

where the traces are replaced by the ensemble averages $\left([\cdot]_{\mathrm{av}}\right)$ with respect to the random vectors; for any operator $\hat{X}, \operatorname{Tr} \hat{X}$ is calculated as

$$
\begin{aligned}
{[\langle\Phi|\hat{X}| \Phi\rangle]_{\mathrm{av}} } & =\sum_{n}\langle n|\hat{X}| n\rangle+\sum_{m, n}\left[\mathrm{e}^{\mathrm{i}\left(\theta_{m}-\theta_{n}\right)}-\delta_{m n}\right]_{\mathrm{av}}\langle n|\hat{X}| m\rangle \\
& \simeq \operatorname{Tr} \hat{X}
\end{aligned}
$$

Finally, $\chi^{\prime \prime}(\omega ; T)$ is obtained by the Fourier transform of $\left\langle M^{x} M^{x}(t)\right\rangle$.

$$
\begin{aligned}
& \chi^{\prime \prime}(\omega ; T)=\left(1-\mathrm{e}^{-\beta \omega}\right) \operatorname{Re} \int_{0}^{\infty}\left\langle M^{x} M^{x}(t)\right\rangle \mathrm{e}^{-\mathrm{i} \omega t} \mathrm{~d} t \\
& =\left(1-\mathrm{e}^{-\beta \omega}\right) \operatorname{Re} \int_{0}^{T_{\max }}\left\langle M^{x} M^{x}(t)\right\rangle \mathrm{e}^{-\mathrm{i} \omega t} \mathrm{e}^{-\eta^{2} t^{2} / 2} \mathrm{~d} t .
\end{aligned}
$$

Here we introduced the Gaussian filter with variance $1 / \eta^{2}$. This $\eta$ determines the frequency resolution. The upper limit of the integral $T_{\max }$ satisfies $T_{\max } \sim 1 / \eta$ in order to avoid the Gibbs oscillation. Also $\eta$ should satisfy the conditions that $0<\eta \ll 1, \eta \ll H$, and $\beta \eta^{2} \ll H$. 


\section{Appendix B: Details of the Triangle Model}

We obtain the block-diagonalized form of the Hamiltonian by using the following basis vectors $\left\{\left|v_{j}\right\rangle\right\}$.

$$
\begin{aligned}
& \left|v_{1}\right\rangle=\frac{-1}{2 \sqrt{3}}[(1+\mathrm{i} \sqrt{3})|\uparrow \uparrow \downarrow\rangle+(1-\mathrm{i} \sqrt{3})|\uparrow \downarrow \uparrow\rangle-2|\downarrow \uparrow \uparrow\rangle], \\
& \left|v_{2}\right\rangle=\frac{-1}{2 \sqrt{3}}[(1-\mathrm{i} \sqrt{3})|\uparrow \uparrow \downarrow\rangle+(1+\mathrm{i} \sqrt{3})|\uparrow \downarrow \uparrow\rangle-2|\downarrow \uparrow \uparrow\rangle], \\
& \left|v_{3}\right\rangle=\frac{-1}{2 \sqrt{3}}[-2|\uparrow \downarrow \downarrow\rangle+(1+\mathrm{i} \sqrt{3})|\downarrow \uparrow \downarrow\rangle+(1-\mathrm{i} \sqrt{3})|\downarrow \downarrow \uparrow\rangle], \\
& \left|v_{4}\right\rangle=\frac{-1}{2 \sqrt{3}}[-2|\uparrow \downarrow \downarrow\rangle+(1-\mathrm{i} \sqrt{3})|\downarrow \uparrow \downarrow\rangle+(1+\mathrm{i} \sqrt{3})|\downarrow \downarrow \uparrow\rangle], \\
& \left|v_{5}\right\rangle=|\uparrow \uparrow \uparrow\rangle, \\
& \left|v_{6}\right\rangle=\frac{1}{\sqrt{3}}[|\uparrow \uparrow \downarrow\rangle+|\uparrow \downarrow \uparrow\rangle+|\downarrow \uparrow \uparrow\rangle], \\
& \left|v_{7}\right\rangle=\frac{1}{\sqrt{3}}[|\uparrow \downarrow \downarrow\rangle+|\downarrow \uparrow \downarrow\rangle+|\downarrow \downarrow \uparrow\rangle], \text { and } \\
& \left|v_{8}\right\rangle=|\downarrow \downarrow \downarrow\rangle .
\end{aligned}
$$

Now we have

$$
\begin{aligned}
& \left\{\begin{array}{l}
\mathcal{H}\left|v_{5}\right\rangle=-\frac{1}{4}(3 J+6 H)\left|v_{5}\right\rangle+\frac{3}{4} D_{+}\left|v_{1}\right\rangle, \\
\mathcal{H}\left|v_{1}\right\rangle=\frac{3}{4} D_{-}\left|v_{5}\right\rangle+\frac{1}{4}\left(3 J-2 H-2 \sqrt{3} D_{z}\right)\left|v_{1}\right\rangle,
\end{array}\right. \\
& \left\{\begin{array}{l}
\mathcal{H}\left|v_{4}\right\rangle=\frac{1}{4}\left(3 J+2 H+2 \sqrt{3} D_{z}\right)\left|v_{4}\right\rangle+\frac{\sqrt{3}}{4} D_{-}\left|v_{6}\right\rangle, \\
\mathcal{H}\left|v_{6}\right\rangle=\frac{\sqrt{3}}{4} D_{+}\left|v_{4}\right\rangle-\frac{1}{4}(3 J+2 H)\left|v_{6}\right\rangle,
\end{array}\right. \\
& \left\{\begin{array}{l}
\mathcal{H}\left|v_{2}\right\rangle=\frac{1}{4}\left(3 J-2 H+2 \sqrt{3} D_{z}\right)\left|v_{2}\right\rangle-\frac{\sqrt{3}}{4} D_{+}\left|v_{7}\right\rangle, \\
\mathcal{H}\left|v_{7}\right\rangle=-\frac{\sqrt{3}}{4} D_{-}\left|v_{2}\right\rangle+\frac{1}{4}(-3 J+2 H)\left|v_{7}\right\rangle,
\end{array}\right. \\
& \left\{\begin{array}{l}
\mathcal{H}\left|v_{8}\right\rangle=\frac{1}{4}(-3 J+6 H)\left|v_{8}\right\rangle+\frac{3}{4} D_{-}\left|v_{3}\right\rangle, \text { and } \\
\mathcal{H}\left|v_{3}\right\rangle=\frac{3}{4} D_{+}\left|v_{8}\right\rangle+\frac{1}{4}\left(3 J+2 H-2 \sqrt{3} D_{z}\right)\left|v_{3}\right\rangle,
\end{array}\right.
\end{aligned}
$$

where $D_{ \pm}=D_{x} \pm \mathrm{i} D_{y}$.

\section{a. Uniaxial Dzyaloshinsky-Moriya interaction}

In the presence of the uniaxial DMI $\left(D_{x}=D_{y}=0\right)$, the triangle-model Hamiltonian is diagonalized by the vectors $\left\{\left|v_{j}\right\rangle\right\}$, i.e., $\left\{\left|v_{j}\right\rangle\right\}$ form the eigenvectors of the Hamiltonian. The 
correspondent eigenvalues $\left\{E_{j}\right\}$ are given by

$$
\begin{aligned}
& E_{8}=-\frac{3}{4} J+\frac{3}{2} H, \\
& E_{7}=-\frac{3}{4} J+\frac{1}{2} H, \\
& E_{6}=-\frac{3}{4} J-\frac{1}{2} H, \\
& E_{5}=-\frac{3}{4} J-\frac{3}{2} H, \\
& E_{4}=\frac{3}{4} J+\frac{1}{2} H+\frac{\sqrt{3}}{2} D_{z}, \\
& E_{3}=\frac{3}{4} J+\frac{1}{2} H-\frac{\sqrt{3}}{2} D_{z}, \\
& E_{2}=\frac{3}{4} J-\frac{1}{2} H+\frac{\sqrt{3}}{2} D_{z}, \text { and } \\
& E_{1}=\frac{3}{4} J-\frac{1}{2} H-\frac{\sqrt{3}}{2} D_{z} .
\end{aligned}
$$

Besides, nonzero matrix elements are obtained as

$$
\begin{aligned}
\left\langle v_{1}\left|M^{x}\right| v_{4}\right\rangle & =-\frac{1}{2}, \\
\left\langle v_{2}\left|M^{x}\right| v_{3}\right\rangle & =-\frac{1}{2}, \\
\left\langle v_{5}\left|M^{x}\right| v_{6}\right\rangle & =\frac{\sqrt{3}}{2}, \\
\left\langle v_{6}\left|M^{x}\right| v_{7}\right\rangle & =1, \text { and } \\
\left\langle v_{7}\left|M^{x}\right| v_{8}\right\rangle & =\frac{\sqrt{3}}{2},
\end{aligned}
$$

where

$$
M^{x}=S_{1}^{x}+S_{2}^{x}+S_{3}^{x} .
$$

Note that $M^{x}$ is hermitian, i.e., $\left\langle v_{i}\left|M^{x}\right| v_{j}\right\rangle=\left(\left\langle v_{j}\left|M^{x}\right| v_{i}\right\rangle\right)^{*}$.

The magnitude relation of $E_{1}, E_{2}$, and $E_{5}$ depends on $H$ :

$$
\left\{\begin{array}{l}
E_{1}<E_{2}<E_{5}\left(H<H_{\mathrm{c}}-\frac{\Delta_{z}}{2}\right), \\
E_{1}<E_{5}<E_{2}\left(H_{\mathrm{c}}-\frac{\Delta_{z}}{2}<H<H_{\mathrm{c}}+\frac{\Delta_{z}}{2}\right), \text { and } \\
E_{5}<E_{1}<E_{2}\left(H_{\mathrm{c}}+\frac{\Delta_{z}}{2}<H\right),
\end{array}\right.
$$

where we define

$$
\Delta_{z} \equiv \sqrt{3} D_{z}
$$


In the absence of the DMI $\left(D_{x}=D_{y}=D_{z}=0\right)$, the two-fold degenerate ground state $\left(\left|v_{1}\right\rangle,\left|v_{2}\right\rangle\right)$ of $M_{z}=1 / 2$ and the state $\left(\left|v_{5}\right\rangle\right)$ of $M_{z}=3 / 2$ intersect at $H=H_{\mathrm{c}}$. In the uniaxial DMI, the states $\left|v_{2}\right\rangle$ and $\left|v_{5}\right\rangle$ intersect at $H=H_{\mathrm{c}}-\Delta_{z}$, and the states $\left|v_{1}\right\rangle$ and $\left|v_{5}\right\rangle$ intersect at $H=H_{\mathrm{c}}+\Delta_{z}$, respectively.

Note that, near $H_{\mathrm{c}}$, we calculate the intensity ratio by taking into account only three low-lying states $\left|v_{1}\right\rangle,\left|v_{2}\right\rangle$, and $\left|v_{5}\right\rangle$ at very low temperatures, and we have

$$
\begin{aligned}
\sum_{m} \mathrm{e}^{-\beta E_{m}} & \simeq \mathrm{e}^{-\beta E_{1}}+\mathrm{e}^{-\beta E_{2}}+\mathrm{e}^{-\beta E_{5}} \\
\sum_{m} r(m) & \simeq r(1)+r(2)+r(5) \\
& \simeq\left(H+\Delta_{z}\right) \mathrm{e}^{-\beta E_{1}}+H \mathrm{e}^{-\beta E_{2}}+3 H \mathrm{e}^{-\beta E_{5}} .
\end{aligned}
$$

Hence $R_{3}(T)$ is approximated as

$$
\begin{aligned}
R_{3}(T) & \simeq \frac{\left(1+\frac{\Delta_{z}}{H}\right) \mathrm{e}^{-\beta E_{1}}+\mathrm{e}^{-\beta E_{2}}+3 \mathrm{e}^{-\beta E_{5}}}{\tanh \left(\frac{\beta H}{2}\right)\left(\mathrm{e}^{-\beta E_{1}}+\mathrm{e}^{-\beta E_{2}}+\mathrm{e}^{-\beta E_{5}}\right)} \\
& \simeq \frac{3+\mathrm{e}^{-\beta\left(H-H_{\mathrm{c}}+\Delta_{z} / 2\right)}+\left(1+\frac{\Delta_{z}}{H}\right) \mathrm{e}^{-\beta\left(H-H_{\mathrm{c}}-\Delta_{z} / 2\right)}}{1+\mathrm{e}^{-\beta\left(H-H_{\mathrm{c}}+\Delta_{z} / 2\right)}+\mathrm{e}^{-\beta\left(H-H_{\mathrm{c}}-\Delta_{z} / 2\right)}} .
\end{aligned}
$$

In the absence of the DMI $\left(\Delta_{z}=0\right)$, we have

$$
R_{3}(T) \simeq \frac{3+2 \mathrm{e}^{-\beta\left(H-H_{\mathrm{c}}\right)}}{1+2 \mathrm{e}^{-\beta\left(H-H_{\mathrm{c}}\right)}} .
$$

Therefore, at ultra-cold temperatures $\left(T \ll\left|H-H_{\mathrm{c}}\right|\right)$, we have

$$
R_{3}(T) \simeq \begin{cases}1+\mathrm{e}^{-\left|H-H_{\mathrm{c}}\right| / T} & \left(H<H_{\mathrm{c}}\right) \\ 3-4 \mathrm{e}^{-\left|H-H_{\mathrm{c}}\right| / T} & \left(H_{\mathrm{c}}<H\right),\end{cases}
$$

and

$$
\frac{\mathrm{d} R_{3}(T)}{\mathrm{d} T} \simeq \begin{cases}\frac{\left|H-H_{\mathrm{c}}\right|}{T^{2}} \mathrm{e}^{-\left|H-H_{\mathrm{c}}\right| / T} & \left(H<H_{\mathrm{c}}\right) \\ -\frac{4\left|H-H_{\mathrm{c}}\right|}{T^{2}} \mathrm{e}^{-\left|H-H_{\mathrm{c}}\right| / T} & \left(H_{\mathrm{c}}<H\right) .\end{cases}
$$

In the case of finite $\Delta_{z}$, at ultra-cold temperatures $\left(T \ll \Delta_{z}\right.$ for $H<H_{\mathrm{c}}-\Delta_{z} / 2$ and $T \ll\left|H-H_{\mathrm{c}}-\Delta_{z} / 2\right|$ for $\left.H_{\mathrm{c}}-\Delta_{z} / 2<H\right)$, we have

$$
R_{3}(T) \simeq \begin{cases}1+\frac{\Delta_{z}}{H}-\frac{2 \Delta_{z}}{H} \mathrm{e}^{-\Delta_{z} / T} & \left(H<H_{\mathrm{c}}-\Delta_{z} / 2\right) \\ 1+\frac{\Delta_{z}}{H}+\left(2-\frac{\Delta_{z}}{H}\right) \mathrm{e}^{-\left|H-H_{\mathrm{c}}-\Delta_{z} / 2\right| / T} & \left(H_{\mathrm{c}}-\Delta_{z} / 2<H<H_{\mathrm{c}}+\Delta_{z} / 2\right) \\ 3-\left(2-\frac{\Delta_{z}}{H}\right) \mathrm{e}^{-\left|H-H_{\mathrm{c}}-\Delta_{z} / 2\right| / T} & \left(H_{\mathrm{c}}+\Delta_{z} / 2<H\right),\end{cases}
$$


and

$$
\frac{\mathrm{d} R_{3}(T)}{\mathrm{d} T} \simeq \begin{cases}-\frac{2 \Delta_{z}^{2}}{T^{2} H} \mathrm{e}^{-\Delta_{z} / T} & \left(H<H_{\mathrm{c}}-\Delta_{z} / 2\right) \\ \frac{1}{T^{2}}\left(2-\frac{\Delta_{z}}{H}\right)\left|H-H_{\mathrm{c}}-\frac{\Delta_{z}}{2}\right| \mathrm{e}^{-\left|H-H_{\mathrm{c}}-\Delta_{z} / 2\right| / T} & \left(H_{\mathrm{c}}-\Delta_{z} / 2<H<H_{\mathrm{c}}+\Delta_{z} / 2\right) \\ \frac{-1}{T^{2}}\left(2-\frac{\Delta_{z}}{H}\right)\left|H-H_{\mathrm{c}}-\frac{\Delta_{z}}{2}\right| \mathrm{e}^{-\left|H-H_{\mathrm{c}}-\Delta_{z} / 2\right| / T} & \left(H_{\mathrm{c}}+\Delta_{z} / 2<H\right) .\end{cases}
$$

In the uniaxial case, the structure of the energy levels is almost the same as that of $V_{15}$ except that the gap at the avoided level crossing point closes. The equation explains the behavior of $R(T)$ in the simulation of $\mathrm{V}_{15}: R^{\prime}(T)<0$ at low fields, $R^{\prime}(T)>0$ at fields close to the avoided level crossing, and $R^{\prime}(T)<0$ at high fields.

\section{b. Weak Dzyaloshinsky-Moriya interaction}

Let us consider the full DMI case $\left(D_{x}=D_{y}=D_{z}=D\right)$. We assume $D>0$ is small in the sense that $D \ll 2\left|H-H_{\mathrm{c}}\right|$ is satisfied.

By ignoring smaller terms than $O(D)$, we have the following eigenvectors $\left\{\left|u_{j}\right\rangle\right\}$.

$$
\begin{aligned}
& \left|u_{1}\right\rangle=-\sqrt{3} \mathrm{e}^{-\mathrm{i} \pi / 4} \xi\left|v_{5}\right\rangle+\left|v_{1}\right\rangle \\
& \left|u_{2}\right\rangle=\left|v_{5}\right\rangle+\sqrt{3} \mathrm{e}^{\mathrm{i} \pi / 4} \xi\left|v_{1}\right\rangle \\
& \left|u_{3}\right\rangle=-\mathrm{e}^{-\mathrm{i} \pi / 4} \eta\left|v_{2}\right\rangle+\left|v_{7}\right\rangle \\
& \left|u_{4}\right\rangle=-\sqrt{3} \mathrm{e}^{\mathrm{i} \pi / 4} \eta\left|v_{8}\right\rangle+\left|v_{3}\right\rangle \\
& \left|u_{5}\right\rangle=\left|v_{2}\right\rangle+\mathrm{e}^{\mathrm{i} \pi / 4} \eta\left|v_{7}\right\rangle \\
& \left|u_{6}\right\rangle=\left|v_{4}\right\rangle-\mathrm{e}^{-\mathrm{i} \pi / 4} \xi\left|v_{6}\right\rangle \\
& \left|u_{7}\right\rangle=\mathrm{e}^{\mathrm{i} \pi / 4} \xi\left|v_{4}\right\rangle+\left|v_{6}\right\rangle \\
& \left|u_{8}\right\rangle=\left|v_{8}\right\rangle+\sqrt{3} \mathrm{e}^{-\mathrm{i} \pi / 4} \eta\left|v_{3}\right\rangle,
\end{aligned}
$$

where

$$
\xi \equiv \frac{-\Delta}{2 \sqrt{2}\left(H-H_{\mathrm{c}}\right)}, \quad \eta \equiv \frac{\Delta}{2 \sqrt{2}\left(H+H_{\mathrm{c}}\right)} .
$$

The corresponding eigenvalues $\left\{E_{j}\right\}$ are given by

$$
\begin{aligned}
& E_{8}=-\frac{3}{4} J+\frac{3}{2} H \\
& E_{7}=-\frac{3}{4} J+\frac{1}{2} H
\end{aligned}
$$




$$
\begin{aligned}
& E_{6}=-\frac{3}{4} J-\frac{1}{2} H \\
& E_{5}=-\frac{3}{4} J-\frac{3}{2} H \\
& E_{4}=\frac{3}{4} J+\frac{1}{2} H+\frac{\Delta}{2} \\
& E_{3}=\frac{3}{4} J+\frac{1}{2} H-\frac{\Delta}{2} \\
& E_{2}=\frac{3}{4} J-\frac{1}{2} H+\frac{\Delta}{2} \\
& E_{1}=\frac{3}{4} J-\frac{1}{2} H-\frac{\Delta}{2} .
\end{aligned}
$$

From the above eigenvectors $\left\{\left|u_{j}\right\rangle\right\}$, nonzero matrix elements are obtained as

$$
\begin{aligned}
& \left\langle u_{1}\left|M^{x}\right| u_{4}\right\rangle=-\frac{1}{2} \\
& \left\langle u_{1}\left|M^{x}\right| u_{6}\right\rangle=-2 \mathrm{e}^{\mathrm{i} \pi / 4} \xi \\
& \left\langle u_{2}\left|M^{x}\right| u_{3}\right\rangle=-\frac{1}{2} \\
& \left\langle u_{2}\left|M^{x}\right| u_{4}\right\rangle=0 \\
& \left\langle u_{2}\left|M^{x}\right| u_{6}\right\rangle=\mathrm{e}^{-\mathrm{i} \pi / 4} \eta \\
& \left\langle u_{2}\left|M^{x}\right| u_{8}\right\rangle=0 \\
& \left\langle u_{3}\left|M^{x}\right| u_{7}\right\rangle=-\eta \mathrm{e}^{-\mathrm{i} \pi / 4} \\
& \left\langle u_{4}\left|M^{x}\right| u_{5}\right\rangle=-\sqrt{3} \mathrm{e}^{\mathrm{i} \pi / 4} \xi \\
& \left\langle u_{4}\left|M^{x}\right| u_{7}\right\rangle=-\mathrm{e}^{\mathrm{i} \pi / 4} \xi \\
& \left\langle u_{5}\left|M^{x}\right| u_{6}\right\rangle=\frac{\sqrt{3}}{2} \\
& \left\langle u_{6}\left|M^{x}\right| u_{7}\right\rangle=1 \\
& \left\langle u_{7}\left|M^{x}\right| u_{8}\right\rangle=\frac{\sqrt{3}}{2} .
\end{aligned}
$$

Let us consider the ultra-cold limit $(\beta \rightarrow \infty)$, where all transitions can be ignored except for the transitions from the ground state. We consider two cases: $H<H_{\mathrm{c}}$, where $\left|v_{1}\right\rangle$ is the ground state, and $H_{\mathrm{c}}<H$, where $\left|v_{5}\right\rangle$ is the ground state. We have

$$
\begin{aligned}
\sum_{m} \mathrm{e}^{-\beta E_{m}} & \simeq \mathrm{e}^{-\beta E_{1}}+\mathrm{e}^{-\beta E_{2}} \\
\sum_{m} r(m) & \simeq r(1)+r(2) \simeq(H+\Delta) \mathrm{e}^{-\beta E_{1}}(H-\Delta) \mathrm{e}^{-\beta E_{2}}
\end{aligned}
$$


for $H<H_{\mathrm{c}}$ and

$$
\begin{aligned}
\sum_{m} \mathrm{e}^{-\beta E_{m}} & \simeq \mathrm{e}^{-\beta E_{5}}+\mathrm{e}^{-\beta E_{1}} \\
\sum_{m} r(m) & \simeq r(5)+r(1) \simeq 3 H \mathrm{e}^{-\beta E_{5}}+(H+\Delta) \mathrm{e}^{-\beta E_{1}}
\end{aligned}
$$

for $H_{\mathrm{c}}<H$. Thus we obtain Eq. (14).

* M. Machida: mmachida@umich.edu

† T. Iitaka: tiitaka@riken.jp

$\ddagger$ S. Miyashita: miyashita@phys.s.u-tokyo.ac.jp

1 A. Müller and J. Döring, Angew. Chem. Int. Ed. Engl. 27, 1721 (1988).

2 A. Müller, J. Döring, and M. Penk, Z. Anorg. Allg. Chem. 595, 251 (1991).

3 D. Gatteschi, L. Pardi, A. L. Barra, A. Müller, and J. Döring, Nature 354, 465 (1991).

4 A. L. Barra, D. Gatteschi, L. Pardi, A. Müller, and J. Döring, J. Am. Chem. Soc. 114, 8509 (1992).

5 A. L. Barra, D. Gatteschi, L. Pardi, A. Müller, and J. Döring, Mol. Eng. 3, 157 (1993).

6 I. Chiorescu, W. Wernsdorfer, A. Müller, H. Bögge, and B. Barbara, Phys. Rev. Lett. 84, 3454 (2000).

7 I. Chiorescu, W. Wernsdorfer, A. Müller, H. Bögge, and B. Barbara, J. Mag. Mag. Mat. 221, $103(2000)$.

8 I. Chiorescu, W. Wernsdorfer, A. Müller, S. Miyashita, and B. Barbara, Phys. Rev. B 67, $020402(2003)$.

9 K. Saito and S. Miyashita, J. Phys. Soc. Jpn. 70, 3385 (2001).

10 I. Dzyaloshinsky, J. Phys. Chem. Solids 4, 241 (1958).

11 T. Moriya, Phys. Rev. Lett. 4, 228 (1960).

12 T. Moriya, Phys. Rev. 120, 91 (1960).

13 S. Miyashita and N. Nagaosa, Prog. Theor. Phys. 106, 533 (2001).

14 S. Miyashita, H. De Raedt, and K. Michielsen, Prog. Theor. Phys. 110, 889 (2003).

15 K. Y. Choi, Y. H. Matsuda, H. Nojiri, U. Kortz, F. Hussain, A. C. Stowe, C. Ramsey, and N. S. Dalal, Phys. Rev. Lett. 96, 107202 (2006).

16 S. Miyashita, S. Tanaka, H. De Raedt, and B. Barbara, J. Phys. Conf. Ser. 143, 012005 (2009). 
17 H. De Raedt, S. Miyashita, and K. Michielsen, Phys. Stat. Sol. B 241, 1180 (2004).

18 H. De Raedt, S. Miyashita, K. Michielsen, and M. Machida, Phys. Rev. B 70, 064401 (2004).

19 M. Machida, T. Iitaka, and S. Miyashita, J. Phys. Soc. Jpn. Suppl. 74, 107 (2005).

20 N. P. Konstantinidis and D. Coffey, Phys. Rev. B 66, 174426 (2002).

21 R. Kubo and K. Tomita, J. Phys. Soc. Jpn. 9, 888 (1954).

22 R. Kubo, J. Phys. Soc. Jpn. 12, 570 (1957).

23 Y. Ajiro, Y. Inagaki, H. Itoh, T. Asano, Y. Narumi, K. Kindo, T. Sakon, H. Nojiri, M. Motokawa, A. Cornia, et al., Physica B 329-333, 1138 (2003).

24 T. Sakon, K. Koyama, M. Motokawa, Y. Ajiro, A. Müller, and B. Barbara, Physica B 346-347, $206(2004)$.

25 F. Chatelin, Valeurs propres de matrices (Eigenvalues of matrices) [in French] (Masson, Paris, 1988).

26 A. Mitsutake, T. Iitaka, and Y. Okamoto, Comp. Phys. Comm. 96, 217 (1996).

27 T. Iitaka, S. Nomura, H. Hirayama, X. Zhao, Y. Aoyagi, and T. Sugano, Phys. Rev. E. 56, 1222 (1997).

28 T. Iitaka and T. Ebisuzaki, Phys. Rev. lett. 90, 047203 (2003).

29 A. Hams and H. De Raedt, Phys. Rev. E 62, 4365 (2000).

30 T. Iitaka and T. Ebisuzaki, Phys. Rev. E 69, 057701 (2004). 